\title{
REFLECTION OF WATER WAVES IN THE PRESENCE OF SURFACE TENSION BY A NEARLY VERTICAL POROUS WALL
}

\author{
A. CHAKRABARTI ${ }^{1}$ and T. SAHOO ${ }^{1}$
}

(Received 29 November 1994; revised 24 January 1996)

\begin{abstract}
In the present paper the problem of reflection of water waves by a nearly vertical porous wall in the presence of surface tension has been investigated. A perturbational approach for the first-order correction has been employed as compared with the corresponding vertical wall problem. A mixed Fourier transform together with the regularity property of the transformed function along the positive real axis has been used to obtain the potential functions along with the reflection coefficients up to first order. Whilst the problem of water of infinite depth is the subject matter of the present paper, a similar approach is applicable to problems associated with water of finite depth.
\end{abstract}

\section{Introduction}

A class of boundary-value problems associated with the two-dimensional Laplace equation appears in the scattering of the linearised theory of water waves involving immersed vertical boundaries. Several methods (see $[2,6,8]$ ) have been considered to solve such boundary-value problems in the literature. Chakrabarti and Sahoo [2] in their recent work have used a method similar to the one considered by Mandal and Kar [6], Mandal and Chakrabarti [5] and Shaw [11] to handle a boundary-value problem which arises in the study of the reflection of water waves by a nearly vertical porous wall. In the present note we have generalised the result of [2] to include the effect of surface tension also. Because of the effect of surface tension, the solution depends on a parameter $\lambda$, known as the edge slope constant (see [10]). By using a perturbational approach which has been discussed by earlier workers, we have converted the problem of concern to two boundary-value problems. We have used a mixed Fourier transform to reduce the Laplace equation to two ordinary differential equations. The solution of one of these two ordinary differential equations is obtained by standard methods whereas the other one is solved by the aid of the Green's function

\footnotetext{
'Department of Mathematics, Indian Institute of Science, Bangalore 560 012, INDIA

(C) Australian Mathematical Society, 1998, Serial-fee code 0334-2700/98
} 
technique. The reflection coefficients up to first order have been determined by using the regularity property of the transformed function on the positive real axis. The particular case of this general problem giving rise to the solution of the vertical wall problem has been derived as a check.

\section{Formulation of the problem}

The problem under consideration is two dimensional in nature. We use a rectangular Cartesian co-ordinate system $(x, y)$, in which the $\mathrm{y}$ axis has been chosen vertically downward with $y=0, x>0$ as the undisturbed mean free surface of water and $x=$ $\epsilon c(y)$ with $y>0$ representing the nearly vertical porous wall, where $\epsilon>0$ is a small parameter and $c(y)$ is a bounded function of $y$ with $c(0)=0$. It is assumed that the fluid in the region $y>0$ is incompressible, the motion is irrotational and simple harmonic in time and is described by the velocity potential $\Phi(x, y, t)=\operatorname{Re}\left[\phi(x, y) e^{-i \omega t}\right]$, " $\operatorname{Re} "$ denoting the "real part", with $\omega$ the angular frequency and $g$ the acceleration due to gravity. We will drop the factor $e^{-i \omega t}$ throughout the rest of this paper. The effect of surface tension on the nearly vertical porous wall will be investigated, by way of determining the velocity potential and the reflection coefficients up to the order of $\epsilon$, where $\phi$ satisfies the partial differential equation

$$
\nabla^{2} \phi=0 \text { in the fluid region, } y>0,
$$

with the boundary conditions

$$
M \phi_{y y y}+\phi_{y}+K \phi=0 \quad \text { on } \quad y=0, x>0 .
$$

Here the positive constants $M$ and $K$ are as given by the relations $M=T / \rho g$ and $K=\omega^{2} / g$ (see $[7,8]$ ) with subscripts denoting partial derivatives, and

$$
\phi_{n}+i k \phi=0 \quad \text { on } \quad x-\epsilon c(y)=0, y>0,
$$

where the subscript $n$ denotes the derivative in the inward normal direction to the boundary $x=\epsilon c(y), k=\frac{b \rho \omega}{\mu}>0$, with $\mu$ being the coefficient of viscosity, $\rho$ the density of fluid and $b$ being a porosity constant, which has the dimension of length. The derivation of the equation (2.3) has been considered by Chakrabarti and Sahoo [2], using a result of Chwang [3]. Also the function $\phi$ has to satisfy the requirements

$$
\text { (I) } \phi,|\nabla \phi| \longrightarrow 0 \text { as } y \longrightarrow \infty
$$

and

$$
\text { (II) } \phi \longrightarrow e^{-k_{0} y-i k_{0} x}+R e^{-k_{0} y+i k_{0} x} \quad \text { as } \quad x \longrightarrow \infty
$$


at infinity, where $R$ is an unknown constant representing the "reflection coefficient", to be determined, $k_{0}$ is the real positive root of the cubic equation $K=\alpha\left(1+M \alpha^{2}\right)$ in $\alpha$. The edge condition to be satisfied by the potential $\phi$ in this case is given by

$$
\phi_{x y}+i k \phi_{y}=\pi \lambda, \quad \text { at } \quad(x, y)=(0+, 0),
$$

in which $\lambda$ is the edge slope constant and is indeterminate in nature (see [10]). The solution of the mathematical problem at hand has ultimately been determined which involves this constant $\lambda$.

Using the Taylor series expansion in the relation (2.3) and considering the terms up to order $\epsilon$ only and neglecting the higher order terms in $\epsilon$, we obtain

$$
\phi_{x}-\epsilon\left(c(y) \phi_{y}\right)_{y}=-i k\left[\phi+\epsilon c(y) \phi_{x}\right] \text { on } \quad x=0 .
$$

Employing a perturbation expansion of the potential $\phi$, the reflection coefficient $R$ and the indeterminable edge slope constant $\lambda$ (as in [2,5 and 6]) in the forms

$$
\begin{aligned}
\phi(x, y, \epsilon) & =\phi_{0}(x, y)+\epsilon \phi_{1}(x, y)+O\left(\epsilon^{2}\right), \\
R & =R_{0}+\epsilon R_{1}+O\left(\epsilon^{2}\right), \\
\lambda & =\lambda_{0}+\epsilon \lambda_{1}+O\left(\epsilon^{2}\right),
\end{aligned}
$$

the problem under consideration reduces to two boundary-value problems for $\phi_{0}$ and $\phi_{1}$, as explained below.

Problem for $\phi_{0}(x, y)$

$$
\nabla^{2} \phi_{0}=0 \quad x>0, y>0
$$

with the boundary conditions

$$
\begin{gathered}
M \phi_{0, y y}+\phi_{0 y}+K \phi_{0}=0 \text { on } y=0, x>0, \\
\phi_{0 x}+i k \phi_{0}=0 \text { on } x=0, \\
\phi_{0},\left|\nabla \phi_{0}\right| \longrightarrow 0 \text { as } y \longrightarrow \infty \\
\phi_{0} \longrightarrow e^{-k_{0} y-i k_{0} x}+R_{0} e^{-k_{0} y+i k_{0}, x} \text { as } x \longrightarrow \infty
\end{gathered}
$$

and

$$
\phi_{0 x y}+i k \phi_{0 y}=\pi \lambda_{0} \quad \text { at } \quad(x, y)=(0+, 0) .
$$


Problem for $\phi_{1}(x, y)$

$$
\nabla^{2} \phi_{1}=0 \text { in the region } x>0, \quad y>0,
$$

with the boundary conditions

$$
\begin{gathered}
M \phi_{1 y y y}+\phi_{1 y}+K \phi_{1}=0 \text { on } y=0, x>0, \\
\phi_{1 x}+i k \phi_{1}=f(y) \text { on } x=0, y>0,
\end{gathered}
$$

where $f(y)=\left[c(y) \phi_{0 y}(0, y)\right]^{\prime}-i k c(y) \phi_{0 x}(0, y)$,

$$
\begin{gathered}
\phi_{1},\left|\nabla \phi_{1}\right| \longrightarrow 0 \text { as } y \longrightarrow \infty, \\
\phi_{1} \longrightarrow R_{1} e^{-k_{0} y+i k_{0} x} \text { as } x \longrightarrow \infty,
\end{gathered}
$$

and

$$
\phi_{1 x y}+i k \phi_{1 y}=\pi \lambda_{1} \quad \text { at } \quad(x, y)=(0+, 0) .
$$

In the next section, we present the method of solution of the above boundary-value problems.

\section{The method of solution}

Determination of $\phi_{0}(x, y) \quad$ Set

$$
\phi_{0}(x, y)=\psi_{0}(x, y)+e^{-k_{0} y-i k_{0} x}+R_{0} e^{-k_{0} y+i k_{0} x}
$$

and use an integral transform of $\psi_{0}(x, y)$ as given by

$$
\hat{\psi}_{0}(\xi, y)=\int_{0}^{\infty} \psi_{0}(x, y)(\xi \cos \xi x-i k \sin \xi x) d x .
$$

Substituting for $\phi_{0}$ in the equation (2.11) with its boundary conditions in the relations (2.12)-(2.16) and transforming the various equations and conditions, we obtain that

$$
\hat{\psi}_{0}^{\prime \prime}-\xi^{2} \hat{\psi}_{0}=A_{0} \xi e^{-k_{0} y},
$$

where a prime denotes an ordinary derivative with respect to $y$ (note that $\xi$ is just a transform parameter), with

$$
\left(1+M \xi^{2}\right) \hat{\psi}_{0}^{\prime}+K \hat{\psi}_{0}+B_{0} M \xi=0 \text { on } \quad y=0,
$$


where $A_{0}=i k_{0}\left(1-R_{0}\right)-i k\left(1+R_{0}\right), B_{0}=\pi \lambda_{0}+i k_{0}\left(k-k_{0}\right)+R_{0} i k_{0}\left(k+k_{0}\right)$, and

$$
\hat{\psi}_{0},\left|\hat{\psi}_{0}^{\prime}\right| \longrightarrow 0, \quad \text { as } \quad y \longrightarrow \infty .
$$

Then, setting

$$
\hat{\psi}_{0}(\xi, y)=P(\xi, y)-\frac{B_{0} M \xi}{K},
$$

we obtain from the equations (3.3), (3.4) and (3.5), the ordinary differential equation

$$
P^{\prime \prime}-\xi^{2} P=\xi A_{0} e^{-k_{0} y}-\frac{\xi^{3} B_{0} M}{K}
$$

with the boundary conditions

$$
\left(1+M \xi^{2}\right) P^{\prime}+K P=0 \quad \text { on } \quad y=0
$$

and

$$
P^{\prime} \longrightarrow 0 \text { as } y \longrightarrow \infty \text {. }
$$

The solution of equation (3.7) satisfying the conditions (3.8) and (3.9) is given by

$$
P(\xi, y)=\frac{\pi \lambda_{0} \xi M e^{-\xi y}}{\xi\left(1+M \xi^{2}\right)-K}-\frac{\xi A_{0} e^{-k_{0} y}}{\xi^{2}-k_{0}^{2}}+\frac{\xi B_{0} M}{K},
$$

which gives

$$
\hat{\psi}_{0}(\xi, y)=\frac{\pi \lambda_{0} \xi M e^{-\xi y}}{\xi\left(1+M \xi^{2}\right)-K}-\frac{\xi A_{0} e^{-k_{0} y}}{\xi^{2}-k_{0}^{2}}
$$

Here we observe that the expression (3.11) for $\hat{\psi}_{0}(\xi, y)$ involves an unknown constant as well as a singularity at $\xi=k_{0}$ on the positive real axis. In order to determine this constant, we use the fact that $\hat{\psi}_{0}$ is a Fourier transform and cannot have a singularity on the positive real axis. This forces the result that

$$
\lim _{\xi \rightarrow k_{0}}\left(\xi-k_{0}\right) \hat{\psi}_{0}(\xi, y)=0,
$$

so that

$$
A_{0}=\frac{2 \pi \lambda_{0} k_{0} M}{1+3 M k_{0}^{2}}
$$


which implies that

$$
R_{0}=\frac{\left(k_{0}-\frac{b \rho \omega}{\mu}\right)}{\left(k_{0}+\frac{b \rho \omega}{\mu}\right)}+\frac{2 i M k_{0} \pi \lambda_{0}}{\left(1+3 M k_{0}^{2}\right)\left(k_{0}+\frac{b \rho \omega}{\mu}\right)} .
$$

Next, using the inverse transform of $\hat{\psi}_{0}(\xi, y)$, as given by the relation (3.2) (see [12]), we obtain that

$$
\psi_{0}(x, y)=c_{0}(y) e^{-i k x}+\frac{2}{\pi} \int_{0}^{\infty} \frac{\hat{\psi}_{0}(\xi, y)(\xi \cos \xi x-i k \sin \xi x)}{\xi^{2}-k^{2}} d \xi
$$

Here and in later occurrences, singular integrals are understood in the sense of their Cauchy principal values and $c_{0}(y)$ is an arbitrary function of $y$ which has to be determined by using the behaviour of $\psi_{0}(x, y)$ as $x \rightarrow \infty$.

Now writing $(\xi \cos \xi x-i k \sin \xi x)=\frac{1}{2}\left\{(\xi-k) e^{i \xi x}+(\xi+k) e^{-i \xi x}\right\}$ and rotating the contour to the positive imaginary axis for the integral involving $e^{i \xi x}$ and to the negative imaginary axis for the integral involving $e^{-i \xi x}$, we can write (3.15) as

$$
\begin{aligned}
\psi_{0}(x, y)= & c_{0}(y) e^{-i k x}+i \hat{\psi}_{0}(k, y) e^{-i k x} \\
& +\frac{2}{\pi} \int_{0}^{\infty} e^{-\eta x} A_{0}(\eta)\left\{\eta\left(1-M \eta^{2}\right) \cos \eta y-K \sin \eta y\right\} d \eta,
\end{aligned}
$$

where

$$
A_{0}(\eta)=\frac{\pi \lambda_{0} M \eta}{(\eta-i k)\left\{K^{2}+\eta^{2}\left(1-M \eta^{2}\right)^{2}\right\}}
$$

Using the behaviour of $\psi_{0}(x, y)$ at $x=\infty$, we obtain

$$
c_{0}(y)=-i \hat{\psi}_{0}(k, y)
$$

Thus

$$
\psi_{0}(x, y)=\frac{2}{\pi} \int_{0}^{\infty} e^{-\eta x} A_{0}(\eta)\left\{\eta\left(1-M \eta^{2}\right) \cos \eta y-K \sin \eta y\right\} d \eta
$$

where $A_{0}(\eta)$ is as given above in (3.17).

The function $\phi_{0}(x, y)$ can finally be determined by $(3.1)$, where $\psi_{0}(x, y)$ and $R_{0}$ are given by (3.19) and (3.14) respectively with $k=b \rho \omega / \mu$. This completes the solution of the problem in this case. 
Determination of $\phi_{1}(x, y) \quad$ We set

$$
\phi_{1}(x, y)=\psi_{1}(x, y)+R_{1} e^{-k_{0} y+i k_{0} x}
$$

Then using a transform as defined by (3.2) for $\psi_{1}(x, y),(2.17)$ with the boundary conditions $(2.18)$ to $(2.22)$ reduce to

$$
\hat{\psi}_{1}^{\prime \prime}-\xi^{2} \hat{\psi}_{1}=\xi\left[A_{1} e^{-k_{0} y}+f(y)\right]-\frac{\xi^{3} B_{1} M}{K},
$$

where $A_{1}=-i\left(k+k_{0}\right) R_{1}$ and $B_{1}=\pi \lambda_{1}+i k_{0} R_{1}\left(k+k_{0}\right)$, with the boundary conditions

$$
\left(1+M \xi^{2}\right) \hat{\psi}_{1}^{\prime}+K \hat{\psi}_{1}=0 \text { on } \quad y=0
$$

and

$$
\hat{\psi}_{1}^{\prime} \longrightarrow 0 \text { as } y \longrightarrow \infty .
$$

In this case we will use a Green's function technique to solve the ordinary differential equation (3.21) in $\hat{\psi}_{1}$ satisfying the boundary conditions (3.22) and (3.23). The solution is given by

$$
\hat{\psi}_{1}(\xi, y)=\xi \int_{0}^{\infty} G(y, s)\left\{A_{1} e^{-k_{0} s}+f(s)-\frac{\xi^{2} B_{1} M}{K}\right\} d s-\frac{\xi B_{1} M}{K},
$$

where $G(y, s)$ is given by

$$
G(y, s)= \begin{cases}\frac{\xi\left(1+M \xi^{2}\right) \cosh \xi s-K \sinh \xi s}{\left\{\xi\left(1+M \xi^{2}\right)-K\right\} \xi} e^{-\xi y} & \text { for } \quad y \geq s \\ \frac{\xi\left(1+M \xi^{2}\right) \cosh \xi y-K \sinh \xi y}{\left\{\xi\left(1+M \xi^{2}\right)-K\right\} \xi} e^{-\xi s} & \text { for } \quad y \leq s .\end{cases}
$$

The expression (3.24) for $\hat{\psi}_{1}(\xi, y)$ has a singularity at $\xi=k_{0}$ which suggests that as in the problem for $\hat{\psi}_{0}$, we must have in this case

$$
\lim _{\xi \rightarrow k_{0}}\left(\xi-k_{0}\right) \hat{\psi}_{1}(\xi, y)=0 .
$$

Using the result

$$
k_{0}\left(1+M k_{0}^{2}\right) \cosh k_{0} y-K \sinh k_{0} y=K e^{-k_{0} y}
$$

we obtain from (3.24) and (3.26) that

$$
A_{1}=\frac{2\left[k_{0} M B_{1}-K a\left(k_{0}\right)\right]}{\left(1+M k_{0}^{2}\right)},
$$


where $a\left(k_{0}\right)=v \int_{0}^{\infty} u(s) e^{-k_{0} s} d s$.

The relation (3.27) for $A_{1}$ implies that

$$
R_{1}=-\frac{2 i\left[K a\left(k_{0}\right)-\pi k_{0} M \lambda_{1}\right]}{\left(k_{0}+b \rho \omega / \mu\right)\left(1+3 M k_{0}^{2}\right)} .
$$

In this case also by Fourier sine inversion

$$
\psi_{1}(x, y)=c_{1}(y) e^{-i k x}+\frac{2}{\pi} \int_{0}^{\infty} \frac{\hat{\psi}_{1}(\xi, y)(\xi \cos \xi x-i k \sin \xi x)}{\xi^{2}-k^{2}} d \xi
$$

with $c_{1}(y)$ an arbitrary unknown function of $y$ which has to be determined by using the condition on $\hat{\psi}_{1}$ as $x \rightarrow \infty$.

Proceeding as in the previous case we obtain the alternate expression

$$
\begin{aligned}
\psi_{1}(x, y)= & c_{1}(y) e^{-i k x}+i \hat{\psi}_{1}(k, y) e^{-i k x} \\
& -\frac{2}{\pi} \int_{0}^{\infty} \frac{e^{-\eta x} A_{1}(\eta)\left\{\eta\left(1-M \eta^{2}\right) \cos \eta y-i K \sin \eta y\right\} d \eta}{(\eta-i k)\left\{K^{2}+\eta^{2}\left(1-M \eta^{2}\right)^{2}\right\}}
\end{aligned}
$$

for $\psi_{1}(x, y)$, where $A_{1}(\eta)$ is given by

$$
A_{1}(\eta)=-M \eta \pi \lambda_{1}+\int_{0}^{\infty} f(s)\left\{\eta\left(1-M \eta^{2}\right) \cos \eta s-K \sin \eta s\right\} d s .
$$

Using the condition on $\psi_{1}$ as $x$ approaches infinity, we obtain that

$$
c_{1}(y)=-i \hat{\psi}_{1}(k, y) \text {. }
$$

Thus, from (3.20), (3.30) and (3.32), we obtain the complete solution

$$
\phi_{1}(x, y)=R_{1} e^{-k_{0} y+i k_{0} x}-\frac{2}{\pi} \int_{0}^{\infty} e^{-\eta x} A_{1}(\eta) \frac{\left\{\eta\left(1-M \eta^{2}\right) \cos \eta y-K \sin \eta y\right\}}{(\eta-i k)\left\{K^{2}+\eta^{2}\left(1-M \eta^{2}\right)^{2}\right\}} d \eta
$$

where $R_{1}$ and $A_{1}(\eta)$ are given by (3.28) and (3.31).

\section{Particular cases}

Case 1 When surface tension is neglected, we have $M=0$ and $k_{0}=K$. Then

$$
\begin{aligned}
& \phi_{0}(x, y)=e^{-K y-i K x}+R_{0} e^{-K y+i K x}, \\
& \phi_{1}(x, y)=R_{1} e^{-K y+i K x}+\int_{0}^{\infty} e^{-\xi x} A_{1}(\xi)(\xi \cos \xi y-K \sin \xi y) d \xi,
\end{aligned}
$$


where

$$
\begin{gathered}
A_{1}(\xi)=\frac{2}{\pi} \frac{\int_{0}^{\infty}(\xi \cos \xi s-K \sin \xi s) f(s) d s}{(-\xi+i b \rho \omega / \mu)\left(\xi^{2}+K^{2}\right)} \\
R_{0}=\frac{K-b \rho \omega / \mu}{K+b \rho \omega / \mu}
\end{gathered}
$$

and

$$
R_{1}=\frac{-2 i K \int_{0}^{\infty} e^{-K s} f(s) d s}{K+b \rho \omega / \mu}
$$

which agrees with the result obtained by Chakrabarti and Sahoo [2].

Case 2 When porosity is neglected and the wall is vertical we have $b \rho \omega / \mu=0$ and $\epsilon=0$. So we get

$$
\begin{aligned}
\phi(x, y)= & \frac{2 \pi i}{1+3 M k_{0}^{2}}\left(A e^{i k_{0} x}+B e^{-i k_{0} x}\right) e^{-k_{0} y} \\
& +2 M \lambda \int_{0}^{\infty} \frac{\left(\xi\left(1-M \xi^{2}\right) \cos \xi y-K \sin \xi y\right) e^{-\xi x}}{\xi^{2}\left(1-M \xi^{2}\right)^{2}+K^{2}} d \xi
\end{aligned}
$$

with $A=\left(1+3 M k_{0}^{2}\right) / 2 \pi i$ and $B=A+M \lambda$. This result agrees with that obtained by Rhodes-Robinson [9] modulo a misprint.

\section{Acknowledgement}

The suggestion made by the referees to bring the paper to this form is gratefully acknowledged. One of the authors (TS) acknowledges the University Grants Commission, New Delhi, for the financial support received as a research student of Indian Institute of Science, Bangalore.

\section{References}

[1] A. Chakrabarti, "A note on the porous-wavemaker problem", Acta Mechanica 77 (1989) 121-129.

[2] A. Chakrabarti and T. Sahoo, "Reflection of water waves by a nearly vertical porous wall", J. Aust. Math. Soc. Series. B 37 (1996), 417-429.

[3] A. T. Chwang, "A porous wavemaker theory", J. Fluid Mechanics 132 (1983) 395-406.

[4] P. J. Kachoyan and W. D. McKee, "Wave forces on steeply sloping sea walls", J. Engin. Maths. 19 (1985) 351-362.

[5] B. N. Mandal and A. Chakrabarti, "A note on diffraction of water waves by a nearly vertical barrier", IMA J. Appl. Maths. 43 (1989) 157-165. 
[6] B. N. Mandal and S. K. Kar, "Reflection of water waves by a nearly vertical wall", Int. J. Math. Educ. Science and Technology 23 (1992) 665-670.

[7] B. A. Packham, "Capillary gravity waves against a vertical cliff”, Proc. Camb. Phil. Soc. 64 (1968) 833-847.

[8] P. F. Rhodes-Robinson, "On surface waves in the presence of immersed vertical boundaries I", $Q$. J. Mech. Appl. Math. 32 (1979) 109-124.

[9] P. F. Rhodes-Robinson, "Note on the reflection of water waves at a wall in the presence of surface tension", Proc. Camb. Phil. Soc. 92 (1982) 369-373.

[10] P. F. Rhodes-Robinson, "On waves in the presence of vertical porous boundaries", J. Aust. Math. Soc. Ser. B (1996), 39 (1997), 104-120.

[11] D. S. Shaw, "Perturbational results for diffraction of water waves by nearly vertical barriers", IMA J. Appl. Math. 33 (1985) 99-117.

[12] 1. N. Sneddon, The Use of Integral Transforms (Tata McGraw Hill, New Delhi, 1974). 